\section{Influence of Stereoregularity on the Wall Slip Phenomenon in Polypropylene}

\section{Prashant S. Tapadia, ${ }^{\dagger}$ Yogesh M. J oshi, ${ }^{\dagger}$ Ashish K. Lele,*,t and R. A. Mashelkar‡}

Chemical Engineering Division, National Chemical Laboratory, Pune 411 008, India, and Council of Scientific and Industrial Research, Anusandhan Bhavan,

2 Rafi Marg, New Delhi 110 001, India

Received August 30, 1999

Introduction. The phenomenon of wall slip occurring during extrusion of certain polymer melts has been extensively investigated for several decades. The history of research on wall slip and the physical mechanisms of slip have been recently reviewed by Wang. ${ }^{1}$ It is generally accepted that for walls of high adhesive energy slip occurs by sudden disentanglement of bulk chains from the adsorbed chains at a critical stress, 2,3 while for walls of low adhesive energy slip occurs by sudden debonding of the adsorbed chains. ${ }^{4}$ We have recently proposed a unified model for wall slip that combines the two mechanisms of slip into one mathematical framework. ${ }^{5}$ Thus, although a lot is now known about the mechanisms of the interfacial slip, it is still not clear as to how the subtleties of molecular architecture influence the slip behavior. Thus, the effects of molecular structural parameters such as molecular weight, molecular weight distribution, tacticity, short- and longchain branching, and the branching distribution are still not completely understood. In this work we show for the first time that the tacticity of polymer chains can significantly influence the slip behavior. We show that syndi otactic polypropylene shows sharkskin distortions on the extrudate surface, which is an indication of wall slip, while an isotactic polypropylene of similar molecular weight and molecular weight distribution does not show any wall slip.

Experimental Section. Four grades of metallocene catalyzed syndiotactic polypropylene (m-sPP) and two grades of metal locene catalyzed isotactic polypropylene (m-iPP) were supplied by the FINA Oil and Chemical Co., USA. One grade of conventional Zeiglar-Natta catalyzed isotactic polypropylene was obtained from Indian Petrochemicals Company Ltd., India. The relevant characteristics of the polypropylene grades used in this work are reported in Table 1.

All extrusion experiments were done on a CEAST Rheovis 2100 rate controlled capillary rheometer. Isotactic polypropylene grades were extruded at $190^{\circ}$, while syndiotactic polypropylene grades were extruded at temperatures of 190 and $140^{\circ} \mathrm{C}$. The PP samples were extruded through case hardened steel dies of $1.0 \mathrm{~mm}$ diameter and L/D of 20 and 30. The dies had an entry angle of $60^{\circ}$. Some experiments were also done using flat entry dies. In some other experiments the internal surface of the dies were modified by coating them with a fluoroelastomer (SUMMIT, Sumitomo Corp., J apan). Coating was done following the procedure recommended by Wang and Drda. ${ }^{6}$ A $5 \%$ solution of the fluoroelastomer in acetone was made to flow through the capillary

† National Chemical Laboratory.

* Council of Scientific and Industrial Research.

* Corresponding author: E-mail lele@che.ncl.res.in.
Table 1. Molecular Parameters of Syndiotactic and Isotactic Polypropylenes

\begin{tabular}{lrcc}
\hline material & \multicolumn{1}{c}{$\mathrm{M}_{\mathrm{w}}$} & $\mathrm{M}_{\mathrm{w}} / \mathrm{M}_{\mathrm{n}}$ & $\begin{array}{c}\text { power law } \\
\text { index }^{13}\end{array}$ \\
\hline m-sPP-1 & 160000 & 4.5 & 0.32 \\
m-sPP-2 & 120000 & 4.1 & 0.34 \\
m-sPP-3 & 115000 & 3.6 & 0.35 \\
m-sPP-4 & 87000 & 3.4 & 0.44 \\
m-iPP-1 & 203000 & 2.5 & 0.36 \\
m-iPP-2 & 160000 & 2.8 & 0.41 \\
ZN-iPP-1 & & $>10$ & 0.36
\end{tabular}

for several times followed by drying the capillary at 220 ${ }^{\circ} \mathrm{C}$ for $12 \mathrm{~h}$

Results and Discussion. Figure 1 shows the photomicrograph of the extrudate surfaces of m-sPP-1 and m-iPP-2 extruded at shear rates of 50, 600, and 2000 $\mathrm{s}^{-1}$. All samples were extruded at $190^{\circ} \mathrm{C}$ using a die of $60^{\circ}$ conical entry angle unless otherwise mentioned. The extrudate of m-sPP-1 at $50 \mathrm{~s}^{-1}$ shows a smooth surface, while that at $600 \mathrm{~s}^{-1}$ shows a typical sharkskin surface similar to that seen in LLDPE. Gross melt fracture was seen at the shear rate of $2000 \mathrm{~s}^{-1}$. All the four grades of m-sPP showed sharkskin above a critical shear stress. Interestingly, none of the grades showed a distinct stick-slip phenomenon or flow oscillations. I mportantly, Figure 1 shows that isotactic polypropylene (m-iPP-2) of the same molecular weight as M-sPP-1 and of similar molecular weight distribution does not show sharkskin distortions on the extrudate surfaces. In fact, none of the iPP grades of high or low molecular weights and of broad or narrow molecular weight distributions showed sharkskin extrudates over the entire range of shear rates studied.

Figure 2 shows the flow curves for all the four grades of m-sPP. It can be seen that the shear rate at which the sharkskin was first seen increases as the molecular weight decreases from m-sPP-1 to m-sPP-4. However, sharkskin was seen at the same shear stress of about $0.2 \mathrm{MPa}$ for the three high molecular weight resins m-sPP-1 to m-sPP-3. F or the m-sPP-4, weak sharkskin distortion was seen at much higher shear stresses and was not as predominant as for the higher molecular weight grades. Extrusion of high molecular weight $\mathrm{m}$-sPP at a lower temperature of $140{ }^{\circ} \mathrm{C}$ showed that the sharkskin appeared again at a shear stress of around $0.2 \mathrm{MPa}$ (and consequently at lower shear rates).

None of the shear rate-shear stress plots showed any nonmonotonic behavior such as that seen typically in the case of a strong stick-slip transition. At higher shear stresses the extrudate surface of the syndiotactic as well as the isotactic polypropylene samples showed gross spiral distortions as seen in Figure 1. We believe that this distortion is due to entry flow instabilities at high flow rates. When the syndi otactic polypropylene samples were extruded through a fluoroelastomer coated die under identical conditions, it was found that while the sharkskin disappeared and the extruded surface became smooth, the spiral gross distortion continued to occur at higher pressure drop. This confirms that the gross distortions are entry instabilities, while the sharkskin defects are indeed interfacial phenomena at the die wall. Also, when a flat entry die was used during extrusion, the gross distortions occurred at a shear rate lower than that for a conical entry die. 

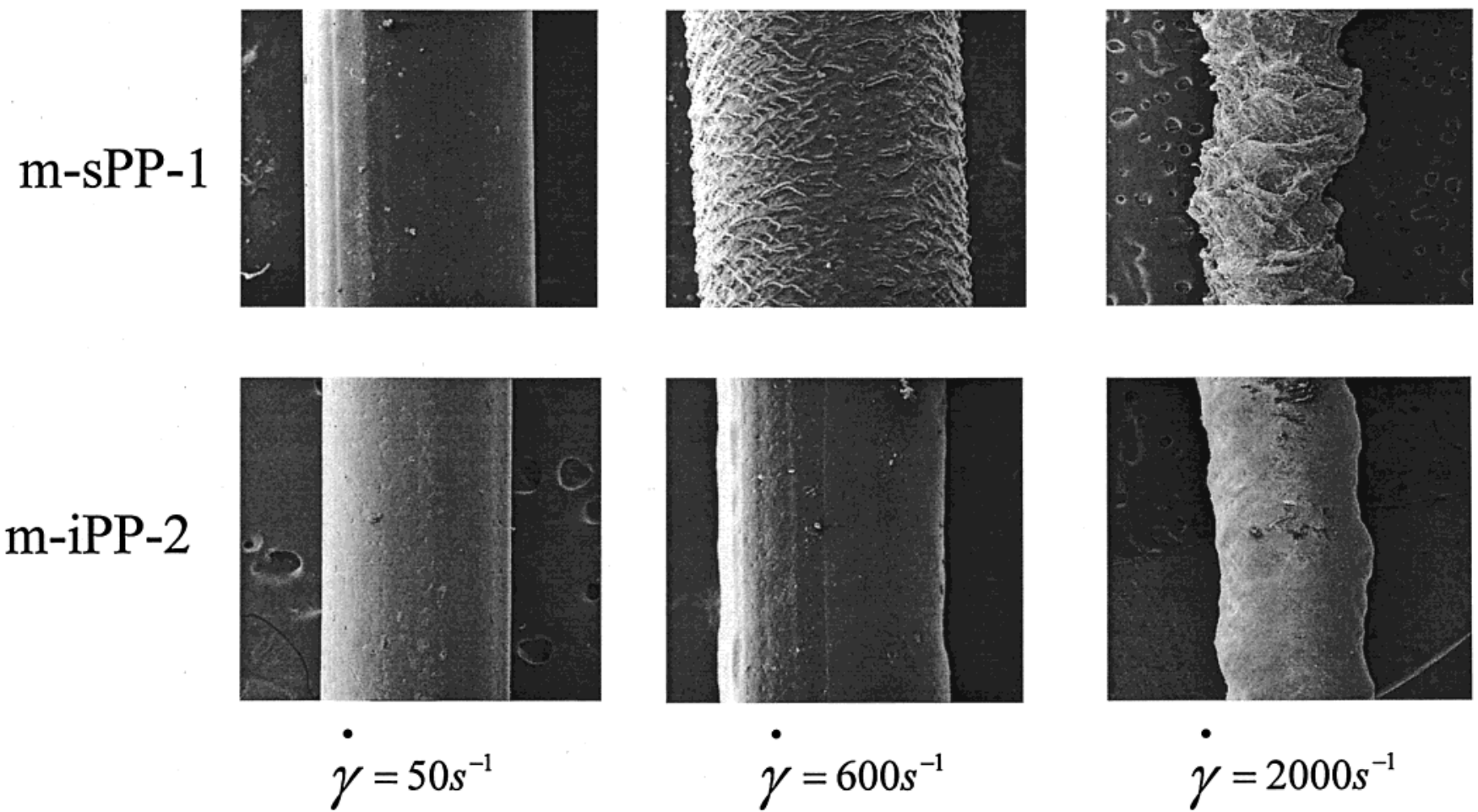

Figure 1. Scanning electron micrographs of extrudates of $\mathrm{m}-\mathrm{SPP}-1\left(\mathrm{M}_{\mathrm{w}}=160000\right)$ and $\mathrm{m}$-iPP-2 $\left(\mathrm{M}_{\mathrm{w}}=160000\right)$ at $190{ }^{\circ} \mathrm{C}$. The m-sPP-1 extrudate shows sharkskin, which is absent in m-iPP-2 of similar molecular weight extruded.

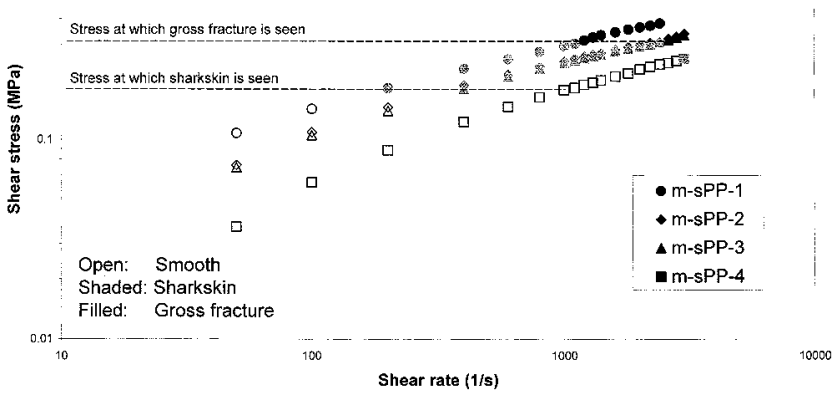

Figure 2. Shear rate-shear stress flow curves of four metallocene SPP's extruded at $190^{\circ} \mathrm{C}, \mathrm{L} / \mathrm{D}=20, \mathrm{D}=1.0 \mathrm{~mm}$ with die entry angle $60^{\circ}$ (open, smooth extrudates; shaded, sharkskin extrudates; filled, gross fracture).

It has been proposed that the sharkskin distortions occur as a result of local interfacial molecular instability (IMI) at the die lip where the stresses build up due to changes in the velocity profile as the extrudate emerges out from the die. ${ }^{7}$ As a result, polymer chains adsorbed on the die wall undergo a coil to stretch transition at a critical shear stress and disentangle from the bulk chains. Consequently, a reduction of the local stress at the die lip allows the chains to recoil and re-entangle with the bulk chains. This reversible transition results in a sharkskin distortion on the extrudate surface. The coil-to-stretch transition of chains and the associated flow instabilities at the die wall can be better understood using the Doi-Edwards tube model as extended by McLeash and Ball. ${ }^{8}$ However, the McLeash and Ball model considered the flow instabilities for the bulk, whereas slip is an interfacial phenomenon. We propose that the MCLeash and Ball model can be applied for tethered chains by using the modified reptation time as suggested by Grassley. ${ }^{9}$ Figure 3 shows schematic of the nonmonotonic flow curve predicted by the M d eash

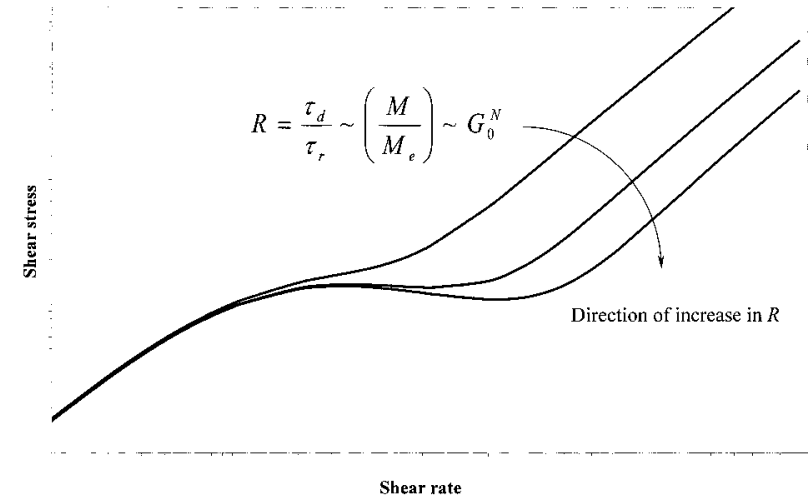

Figure 3. Schematic of the nonmonotonic flow curve predicted by the extended Doi-Edwards theory. It can be seen that as $R\left(\sim G_{0}^{N}\right)$ increases, the tendency of nonmonotonicity increases.

and Ball model. At the point of maximum stress in the nonmonotonic region theadsorbed chains undergo a coilto-stretch transition resulting in slip. The model predicts that nonmonotonic nature of the flow curve depends on the parameter $\mathrm{R}$, which is the ratio of the reptation time to the Rouse relaxation time of the chains. Thus,

$$
\mathrm{R}=\frac{\tau_{\mathrm{d}}}{\tau_{\mathrm{r}}} \sim \frac{\mathrm{M}}{\mathrm{M}_{\mathrm{e}}} \sim \mathrm{G}_{0}^{\mathrm{N}}
$$

where $G_{0}^{N}$ is the plateau modulus, $M$ is the molecular weight, and $\mathrm{M}_{\mathrm{e}}$ is the entanglement molecular weight. 8,10 The greater the value of $\mathrm{R}$, the greater is the nonmonotonic nature of the flow curve. This implies that the materials with the higher plateau modulus or with the lower entanglement molecular weight will have a tendency to slip during extrusion. Syndiotactic polypropylene has a significantly higher plateau modulus of 1.35 $\mathrm{MPa}$ than that of isotactic polypropylene which is 
around $0.42 \mathrm{MPa}$. Equivalently, $\mathrm{M}_{\mathrm{e}}$ for sPP is $\sim 1700$ $2200 \mathrm{~g} / \mathrm{mol}$, which is smaller than that of iPP, 5500$7000 \mathrm{~g} / \mathrm{mol} .11,12$ Thus, sPP has a greater entanglement density than iPP and hence would be more susceptible to a coil-to-stretch transition compared to the case of iPP. Indeed our experiments show that SPP shows wall slip, while iPP does not slip.

It is clear from the above discussion that the adsorbed syndiotactic polypropylene molecules can undergo a coilto-stretch transition at the die lip. However, it is not clear as to why the SPP does not show a global stickslip transition. There can be two probable reasons. It is possible that, before reaching the critical stress level at which a global stick-slip transition would occur, the entry flow instabilities set in, and hence stick-slip is not seen experimentally. The other possibility is that since SPP is not as highly entangled as linear polyethylenes $\left(\mathrm{G}_{0}^{\mathrm{N}} \sim 3 \mathrm{MPa}\right){ }^{12}$ a coil-to-stretch transition would occur only under the influence of the small elongational velocity component that exists at the die lip. The pure shear flow field existing inside the capillary may not be sufficient to stretch the adsorbed SPP chains. Further work is required to understand the effects of inherent chain rigidity and entanglement density on the stick-slip transition.

Our results clearly show the effect of one of the important molecular structural parameters, namely tacticity, on the wall slip behavior of melts. These results are also important from the point of view of processing of commercial SPP grades for films and fiber applications.
Acknowledgment. Wethank Dr. Abbas Razavi, Dr. David Anderson, and Dr. Demain Axel of FINA Oil and Chemical Co., USA, for providing us metallocene polypropylenes. We are also thankful to Dr. Sainkar, of NCL, Pune for the SEM photomicrographs.

\section{References and Notes}

(1) Wang, S. Q. Adv. Polym. Sci. 1999, 138, 227-275.

(2) Brochard, F.; de Gennes, P. G. Langmuir 1992, 8, 30333037.

(3) Wang, S. Q.; Drda, P. A. Macromolecules 1996, 29, 26272632.

(4) Hill, D. A. J . Rheol. 1998, 42, 581-601.

(5) J oshi, Y. M.; Lele, A. K.; Mashelkar, R. A. J . Non-Newtonian Fluid Mech. 1999, 89 (3), 303-335.

(6) Wang, S. Q.; Drda, P. A. Rheol. Acta 1997, 36, 128-134.

(7) Barone, J . R.; Plucktaveesak, N.; Wang, S. Q. J . Rheol. 1998, 42, 813-832.

(8) McLeish, T. C. B.; Ball, R. C. J . Polym. Sci., Part B: Polym. Phys. 1986, 24, 1735-1745.

(9) Graessley, W. W. Adv. Polym. Sci. 1982, 47, 67-117.

(10) Doi, M.; Edwards, S. F. The Theory of Polymer Dynamics; Clarendon Press: Oxford, 1989; p 237.

(11) Eckstein, A.; Suhm, J .; Friedrich, C.; Maier, R. D.; Sassmannshausen, J .; Bochmann, M.; Mulhaupt, R. Macromolecules 1998, 31, 1335-1340.

(12) Fetters, L. J .; Lohse, D. J .; Graessley, W. W. J . Polym. Sci., Part B: Polym. Phys. 1999, 37, 1023-1033.

(13) Power law index " $\mathrm{n}$ " is defined by power law model $\eta=$ $m\left(I_{D}\right)^{(n-1) / 2}$ and describes the shear thinning nature of the fluid. $I_{D}$ is the second invariant of rate of strain tensor.

MA991480L 